\title{
Towards the production of an ultra cold antihydrogen beam with the AEGIS apparatus
}

\author{
James William Storey on behalf of the AEGIS \\ collaboration
}

Published online: 18 October 2011

(C) Springer Science+Business Media B.V. 2011

\begin{abstract}
The AEGIS (Antimatter Experiment: Gravity, Interferometry, Spectroscopy) experiment is an international collaboration, based at CERN, with the experimental goal of performing the first direct measurement of the Earth's gravitational acceleration on antihydrogen. In the first phase of the experiment, a gravity measurement with $1 \%$ precision will be performed by passing a beam of ultra cold antihydrogen atoms through a classical Moiré deflectometer coupled to a position sensitive detector. The key requirements for this measurement are the production of ultra cold ( $\mathrm{T} \sim 100 \mathrm{mK}$ ) Rydberg state antihydrogen and the subsequent Stark acceleration of these atoms. The aim is to produce Rydberg state antihydrogen by means of the charge exchange reaction between ultra cold antiprotons ( $\mathrm{T} \sim 100 \mathrm{mK}$ ) and Rydberg state positronium. This paper will present details of the developments necessary for the successful production of the ultra cold antihydrogen beam, with emphasis on the detector that is required for the development of these techniques. Issues covered will include the detection of antihydrogen production and temperature, as well as detection of the effects of Stark acceleration.
\end{abstract}

Keywords Antihydrogen • Equivalence principal - Scintillator fibre detector

\section{Introduction}

General relativity is a classical theory of physics, for which there is no distinction between matter and antimatter particles. A long held ambition of theoretical physics

AEGIS collaboration, http://aegis.web.cern.ch/aegis.

J. W. Storey $(\otimes)$

Physik-Institut, University of Zurich, Winterthurerstr. 190, 8057 Zurich, Switzerland

e-mail: james.storey@cern.ch 
has been the development of a so called quantum theory of gravity that would combine general relativity and quantum mechanics. Candidate quantum theories of gravity allow that, in principle, antimatter may fall differently from ordinary matter in the Earth's gravitational field. Specifically, theories of supergravity can introduce graviscalar (spin-0) and gravivector (spin-1) components to the gravitational interaction. The gravivector component leads to an attractive force acting between opposite charges which would affect the fall of antimatter particles in the Earth's gravitational field and would be manifest as a violation of the weak equivalence principal (universality of free fall) [1].

A strong motivation to consider the possibility that antimatter presents a negative active gravitational mass comes from the observation in 1998 of an accelerating universe [2,3]. The $\Lambda$-CDM standard model of the universe provides for an excellent parameterisation of the current cosmological data, but requires as yet two unobserved components, dark matter and dark energy, to account for $95 \%$ of the universe. A recently proposed alternative model which requires neither, the so-called "Dirac-Milne" universe, is based on a matter-antimatter symmetric cosmology, in which antimatter provides a negative gravitational mass [4].

The most stringent limits on any asymmetry between the free fall of matter and antimatter comes from the analysis of the Eöt-Wash experiment, an Eötvos type torsion balance experiment using test bodies of different compositions [5]. However, there has never been a successful direct measurement of the behaviour of antimatter in a gravitational field. Earlier attempts at Stanford [6] and at CERN's Low Energy Antiproton Ring [7] were hampered by the overwhelming effect of stray electric and magnetic fields upon the charged test particles.

The production of copious amounts of cold antihydrogen $(\overline{\mathrm{H}})$ by the ATHENA and ATRAP experiments at CERN's Antiproton Decelerator (AD) has opened the possibility to measure the effect of gravity on neutral antimatter $[8,9]$. The AEGIS experiment has been designed to address this important question.

\section{Experimental principle}

The primary scientific goal of AEGIS is the direct measurement of the effect of the Earth's gravitational field on antihydrogen. In the first phase of the experiment the effect of gravity will be measured with a $1 \%$ precision by observing the vertical displacement of the shadow image produced by a beam of antihydrogen atoms which pass through a Moiré deflectometer, the classical counterpart of an atom interferometer. This experiment will represent the first direct measurement of the gravitational attraction of antimatter and matter.

A schematic of the core components of the AEGIS apparatus is shown in Fig. 1. The key experimental challenge is the production of ultra-cold $(<100 \mathrm{mK})$ antihydrogen together with the subsequent acceleration of the anti-atoms to create a beam in the direction of the Moiré deflectometer, and to measure the vertical displacement of the antihydrogen with $10 \mu \mathrm{m}$ precision. 


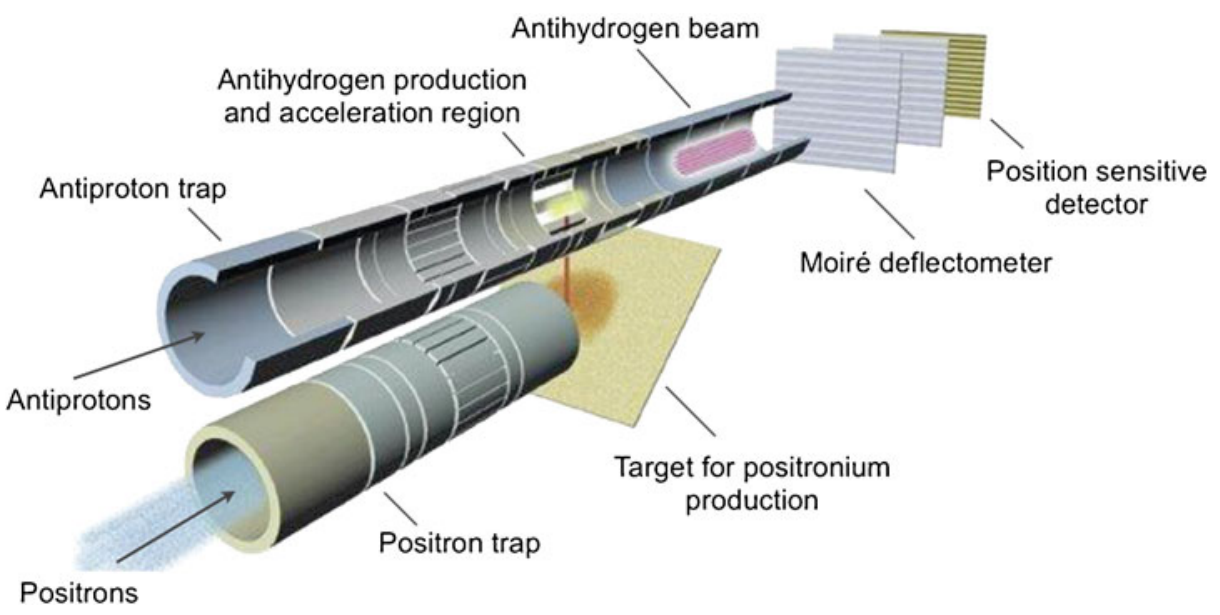

Fig. 1 Schematic of the core components of the AEGIS apparatus, comprising of two parallel Penning-Malmberg traps which are used to trap and manipulate the antiprotons and positrons, and to produce and accelerate the antihydrogen towards a Moiré deflectometer coupled to a position sensitive detector

\section{Production of the antihydrogen beam}

\subsection{Beam requirements}

A measurement of the acceleration of antihydrogen in the Earth's gravitational field, $g_{\overline{\mathrm{H}}}$, is extracted from a parabolic fit to the fringe shift, $\phi$, as a function of flight time, $T$, between the two gratings. The precision of the measurement of $g_{\overline{\mathrm{H}}}$ is expected to be dominated by the uncertainty of the fringe shift measurement $\sigma_{\phi}$. From simulations:

$$
\sigma_{\phi}=\frac{0.8}{\sqrt{N}} \mathrm{rad}
$$

where $N$ is the number of antihydrogen annihilations reconstructed by a free-fall detector with a resolution of $10 \mu \mathrm{m}$. The number of antihydrogen atoms $N$ arriving on a free fall-detector of radius $w$ at a length $l$ from the point where $N_{0}$ antihydrogen are produced with velocity $v_{\mathrm{h}}$ in the direction of the free-fall detector and transverse velocity $v_{\mathrm{t}}$, is:

$$
N=N_{0} \times \frac{w^{2}}{l^{2}} \times \frac{v_{\mathrm{h}}^{2}}{v_{\mathrm{t}}^{2}} \times f_{\text {grating }} \times \epsilon_{\mathrm{det}}
$$

where $f_{\text {grating }}$ is the transmission efficiency through the gratings and $\epsilon_{\text {det }}$ is the efficiency of the free-fall detector to reconstruct the antihydrogen annihilations. For the current AEGIS design where $l=100 \mathrm{~cm}$ and $w=10 \mathrm{~cm}$, it follows from Eq. 2 that $v_{\mathrm{t}}<50 \mathrm{~m} / \mathrm{s}$ in order to ensure that all transmitted antihydrogen atoms are incident on the free fall detector with a vertical displacement of at least $20 \mu \mathrm{m}\left(v_{\mathrm{h}}<500 \mathrm{~m} / \mathrm{s}\right)$. 
Fig. 2 Proposed scheme for the production and acceleration of antihydrogen atoms

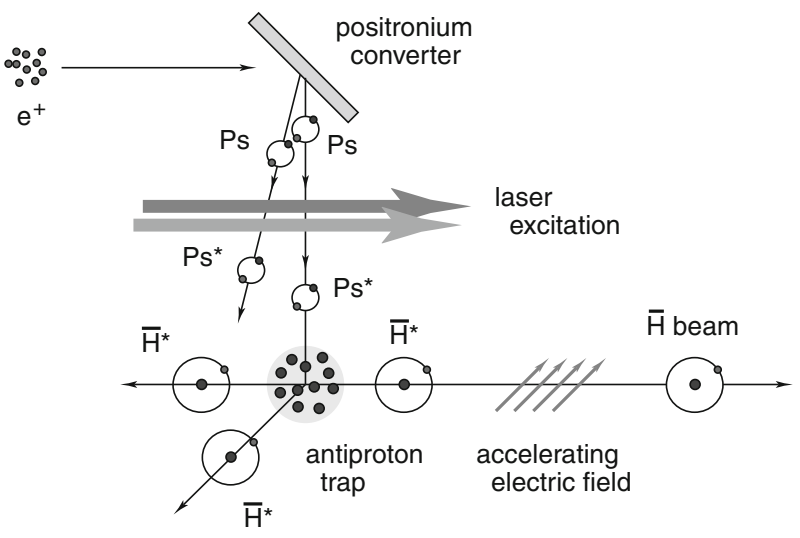

The highly desirable condition that all antihydrogen produced should be incident on the free-fall detector is what motivates the experimentally challenging requirement for the production of ultra-cold $(T<100 \mathrm{mK})$ antihydrogen. However, as shown by Eq. 1, the precision on the fringe shift is a function of the number of detected atoms which in addition to the temperature of the antihydrogen, also depends on the number, $N_{0}$, of antihydrogen atoms produced per experimental cycle, the repetition rate of each experimental cycle and the period of apparatus stability. For example, a measurement of $g_{\overline{\mathrm{H}}}$ with $1 \%$ precision requires $10^{4}$ detected atoms: this requires the production of 30 antihydrogen atoms at $100 \mathrm{mK}$ every 120 s for 2 weeks.

\subsection{Beam production}

The production of antihydrogen by ATHENA and ATRAP employed a nested-well technique to produce antihydrogen [10], the temperature of which is many orders of magnitude greater than that required by AEGIS [11,12]. A promising technique to produce ultra-cold antihyrodgen is based on the charge exchange reaction between antiprotons $(\overline{\mathrm{p}})$ and highly excited Rydberg state positronium (Ps*)

$$
\mathrm{Ps}^{*}+\overline{\mathrm{p}} \rightarrow \overline{\mathrm{H}}^{*}+\mathrm{e}^{-}
$$

in which the bound positron $\left(\mathrm{e}^{+}\right)$is captured by the antiproton and an electron is released $\left(\mathrm{e}^{-}\right)$[13]. In this scheme it is expected that the antihydrogen velocity will be dominated by the temperature of the trapped antiprotons, which will be cooled to sub-Kelvin temperatures by means of electron, resistive and evaporative cooling techniques $[14,15]$. Another benefit of this scheme is the large cross section, which scales to the fourth power of positronium principal quantum number. Furthermore, the production of antihydrogen in an excited state is required for the subsequent acceleration of the anti-atoms in inhomogeneous electric fields.

The principal of the scheme is illustrated in Fig. 2. Antihydrogen production begins with the production of positronium (Ps) by means of the acceleration of $10^{8}$ positrons with a time length of $10-20 \mathrm{~ns}$ from a Surko type accumulator onto a nanoporous material. The ortho-Ps emitted from the target is then brought to the Rydberg state by means of a two-step laser excitation [16]. A fraction of the Rydberg positronium diffuses across a Penning trap region in which $10^{5}$ antiprotons have been 
stored and cooled to $\sim 100 \mathrm{mK}$. Antihydrogen is produced by means of the charge exchange reaction, which is expected to have a large cross section of $\sim 10^{-9} \mathrm{~cm}^{2}$ for positronium with a principal quantum number $(n)$ greater than 30 . Taking into account that the velocity of the Rydberg positronium must be in the order of $10^{4}-10^{5} \mathrm{~m} / \mathrm{s}$ to optimise the charge exchange reaction and the antiproton plasma dimensions must be in the order of a few $\mathrm{mm}$, the antihydrogen is produced in a $1 \mu \mathrm{s}$ time window. This is in contrast to the nested-well techniques used by ATRAP and ATHENA where antihydrogen is produced over a timescale of seconds. For AEGIS the pulsed production scheme has the benefit that the time of the pulse provides a start time $(t=0)$ for the gravity measurement. Pulsed production also has important implications for the detection of antihydrogen production and temperature, which is discussed further in Section 4.1.

The intention is then to create a beam of antihydrogen by means of Stark acceleration, whereby the anti-atoms experience a force when the electric dipole moment is exposed to an electric field gradient. This technique has already been demonstrated by members of the AEGIS collaboration with hydrogen atoms [17]. In these experiments accelerations of $10^{8} \mathrm{~m} / \mathrm{s}^{2}$ were achieved using a pair of electrodes. AEGIS will use a similar field configuration, generated by the electrodes of the Penning trap, to accelerate the formed antihydrogen to approximately $400 \mathrm{~m} / \mathrm{s}$ in the direction of the Moiré deflectometer.

\section{Antihydrogen beam diagnostics}

\subsection{Requirements}

It is anticipated that the complete AEGIS apparatus, less the Moiré deflectometer, will be installed in time for the CERN antiproton decelerator (AD) run in 2012. Objectives in the first phase of the experiment are:

- Demonstrate the production of antihydrogen by means of the charge exchange reaction between Rydberg state positronium and antiprotons.

- Measure the temperature of the antihydrogen produced.

- Demonstrate the production of an antihydrogen beam by means of Stark acceleration.

The principal diagnostic tool to provide measurables for these objectives is the socalled "central detector". In the following section the requirements of this detector are described.

\subsubsection{Detection of antihydrogen production}

The production of cold antihydrogen was first demonstrated by the ATHENA and ATRAP collaborations by means of the spatial-temporal coincidence of the constituent antiproton and positron annihilation (ATHENA) and by identifying the antiproton liberated from an antihydrogen atom that has been field-ionised (ATRAP) [8, 9]. The ATHENA technique is not suitable for the AEGIS experiment 


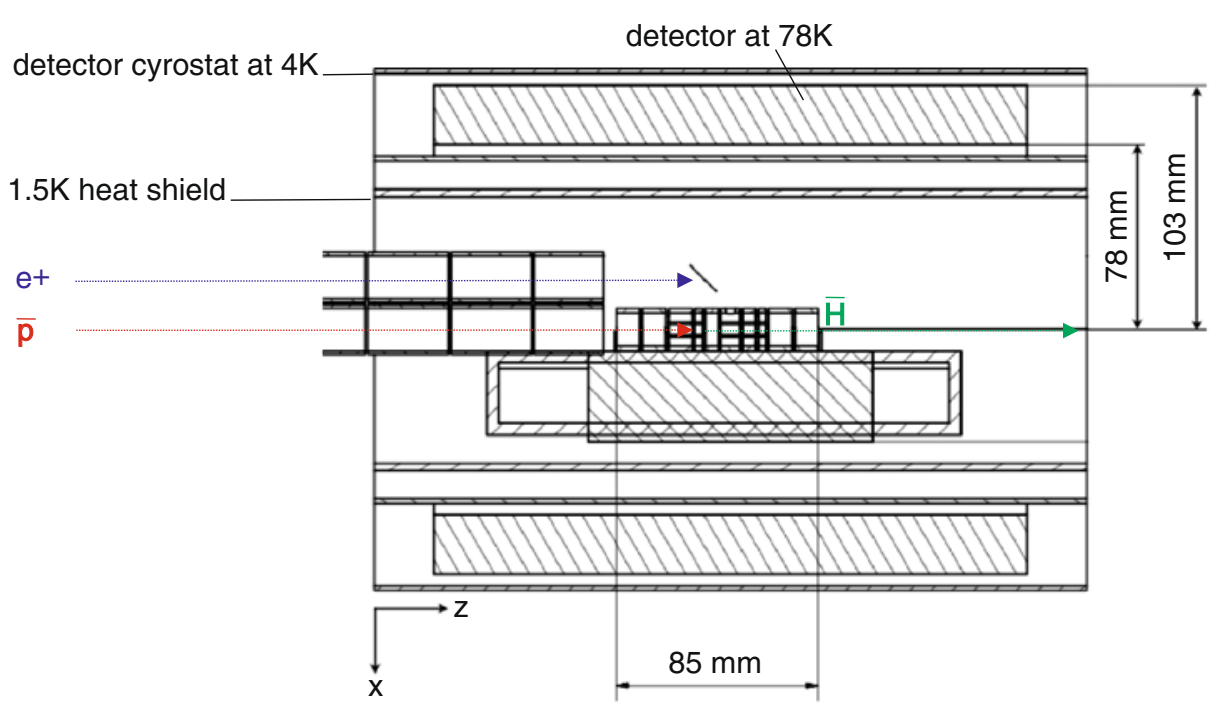

Fig. 3 Schematic of the central detector environment shown in the vertical-beam axis plane (x-z). The location of the space allocated for the central detector is indicated by diagonal lines between $78 \mathrm{~mm}$ and $103 \mathrm{~mm}$ of the beam axis. Also shown are the recombination Penning trap electrodes where antihydrogen will be produced (section indicated by $85 \mathrm{~mm}$ length), the parallel Penning traps which provide the antiprotons and the trap above this which will accelerate positrons onto the positronium target. Vertically below the recombination electrodes is the dilution refrigerator which will thermally anchor these electrodes to $100 \mathrm{mK}$

as the space available for the detector, shown in Fig. 3, is insufficient to allow for both the multi-layered position sensitive detector required to reconstruct the position of the antiproton annihilation and the array of crystals required to identify the backto-back $511 \mathrm{keV} \gamma$ 's from the positron annihilation. Furthermore, the antihydrogen detection efficiency of the ATHENA technique is low due to the typically low $\gamma$ detection efficiency.

A conceptually similar approach to produce antihydrogen using Caesium atoms to produce Rydberg state positronium rather than a nanoporous target has been demonstrated by the ATRAP collaboration using the field-ionisation technique [18]. However, this method suffers from a very low detection efficiency due to the limited field ionisation solid angle, which combined with the expected low antihydrogen yield, implies that this technique is unsuitable for AEGIS.

One promising technique for AEGIS is based on the observation by ATHENA that the spatial distribution of reconstructed annihilations on the Penning trap wall are isotropic for antihydrogen and localised to a hot spot for uncombined antiprotons [19]. In which case three-dimensional reconstruction of the annihilation is required with a resolution of a few millimetres, but no $\gamma$ reconstruction is needed. The AEGIS scheme for pulsed production of antihydrogen also opens the opportunity for new antihydrogen detection methods by, for example, exploiting the relatively short production time compared to the long drift time of the antihydrogen from the point of production to the point of annihilation on the Penning trap wall which is shown in Fig. 4 to be at least a few microseconds. 
Fig. 4 Drift time of the antihydrogen atoms from the time of production $(t=0)$ to the point of annihilation on Penning trap wall for antihydrogen produced with temperatures $T=100 \mathrm{~K}$, $T=10 \mathrm{~K}$ and $T=100 \mathrm{mK}$

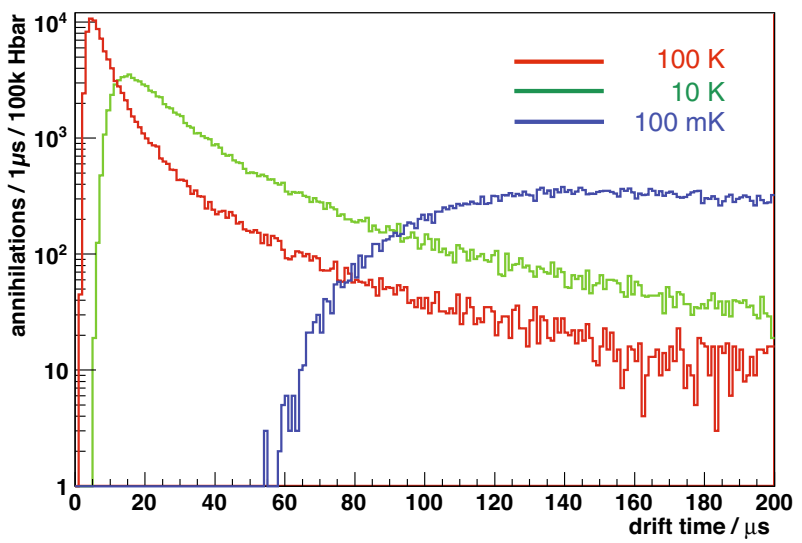

\subsubsection{Temperature measurement and identification of Stark acceleration}

The pulsed production scheme offers the possibility to measure the temperature of the antihydrogen by means of the drift time from the known time of production to the time of the annihilation. A simulation of the drift time is shown in Fig. 4 for antihydrogen with temperature $T=100 \mathrm{~K}, 10 \mathrm{~K}$ and $100 \mathrm{mK}$. Identifying antihydrogen annihilations with a time resolution of the order of a microsecond is sufficient to resolve each of the three distributions.

In order to identify the effects of the Stark acceleration it is necessary to reconstruct the position of the annihilation on the beam axis, but a full three dimensional reconstruction is not required.

\subsubsection{Data acquisition and environment}

Antihydrogen production using the nested-well technique typically yields in the order of 1,000 antihydrogen atoms in a few seconds, with approximately a millisecond between annihilations. In contrast, in the AEGIS pulsed production scheme it is expected that $50 \%$ of $10 \mathrm{~K}$ antihydrogen annihilate less than $500 \mathrm{~ns}$ after the previous annihilation. The detector must therefore be extremely fast, but only needs to be active for approximately a millisecond every $100 \mathrm{~s}$.

The detector must occupy a cylindrical volume centred on the Penning trap used for the charge exchange reaction, with an inner radius of $78 \mathrm{~mm}$ and outer radius of $103 \mathrm{~mm}$. As the detector is located in a cryogenic environment it must operate at $100 \mathrm{~K}$ and produce not more than $10 \mathrm{~W}$ of heat. An additional complication is the $\sim 1$ billion $511 \mathrm{keV} \gamma$ 's which are produced inside the detector volume when the positrons are accelerated onto the positronium target, which occurs only a few microseconds before the first annihilations.

\subsection{Scintillating fibre detector with silicon photomultiplier readout}

Considering the requirements presented in the previous section it has been decided to construct a detector that is capable of resolving the location of the antiproton annihilation spatially and temporally. The detector will consist of three layers of $1 \mathrm{~mm}$ diameter Kuraray SCSF-78M multi-clad scintillating fibres coupled to clear 
fibres of the same diameter which will direct the optical signal from the cryogenic region onto arrays of $1 \mathrm{~mm}$ diameter silicon photomultipliers. Each layer will consist of orthogonal helical fibres wound in the direction of the beam axis. A prototype design is currently under construction to validate the mechanical aspects of the fibres, glue, connectors and support structure at cryogenic temperatures.

\section{Summary and outlook}

The AEGIS experiment is designed to measure the acceleration of antihydrogen in the Earth's gravitational field. If successful, this experiment will be the first direct measurement of the gravitational attraction of antimatter and matter. The key experimental challenge will be the production and acceleration of $100 \mathrm{mK}$ antihydrogen. A detector based on scintillating fibres coupled to silicon photomultipliers is being developed to detect the production, temperature and acceleration of the antihydrogen atoms, information from which will be crucial in developing the methods to produce the required antihydrogen beam. The experiment is currently under construction at the CERN AD and will be ready in 2012 for the first attempts at producing an antihydrogen beam.

\section{References}

1. Sherk, J.: Phys. Lett. B 88, 265 (1979)

2. Riess, A.G., et al.: Astron. J. 116, 1009 (1998)

3. Perlmutter, S., et al.: Astrophys. J. 517, 565 (1999)

4. Benoit-Lévy, A., Chardin, G.: arXiv:0903.2446v1 (2009). Accessed 13 March 2009

5. Alves, D., Jankowiak, M., Saraswat, P.: arXiv:0907.4110v1 (2009). Accessed 23 July 2009

6. Fairbank, W.M., et al.: In: Bertotti, B. (ed.) Experimental Gravitation, p. 310. Academic, New York (1974)

7. Holzscheiter, M.H., et al.: Nucl. Phys. A 558, 709c (1993)

8. Amoretti, M., et al.: Nature 419, 456 (2002)

9. Gabrielse, G., et al.: Phys. Rev. Lett. 89, 213401 (2002)

10. Gabrielse, G., Rolston, S.L., Haarsma, L., Kells, W.: Phys. Lett. A 129, 38 (1988)

11. Madsen, N., et al.: Phys. Rev. Lett. 94, 033403 (2005)

12. Gabrielse, G., et al.: Phys. Rev. Lett. 93, 073401 (2004)

13. Charlton, M.: Phys. Lett. A 143, 143 (1990)

14. Gabrielse, G., et al.: Phys. Rev. Lett. 63,1360 (1989)

15. Andresen, G.B., et al.: Phys. Rev. Lett. 105, 013003 (2010)

16. Giammarchi, M.G., et al.: Hyperfine Interact. 193, 321 (2009)

17. Vliegen, E., Merkt, F.: J. Phys. B 39, 241 (2006)

18. Storry, C.H., et al.: PRL 93, 263401 (2004)

19. Fujiwara, M.C., et al.: Phys. Rev. Lett. 92, 065005 (2004) 